Pacific Journal of Mathematics

AN IDENTITY FOR MATRIX FUNCTIONS 


\title{
AN IDENTITY FOR MATRIX FUNCTIONS
}

\author{
RUSSELL MERRIS
}

Let $G \subset S_{n}$. Let $\eta$ be a character on $G$. For $A=\left(a_{i j}\right)$ an $n$-square matrix, define

$$
d_{\eta}^{Q}(A)=\sum_{g \in G} \eta(g) \prod_{t=1}^{n} \alpha_{t g(t)} .
$$

A general identity for idempotents in group algebras is proved. A very special example of the consequences is this: If $\chi$ is a linear character on $G$ and $H$ a normal subgroup of $G$, then $[G: H] d_{\chi}^{H}(A)=\sum \eta(1) d_{\eta}^{G}(A)$, where the summation is over those irreducible characters $\eta$ of $G$ whose restriction to $H$ contain $\chi$ as a component.

1. Introduction. In this note we prove two theorems involving idempotents in group algebras. The first is a very general identity between the central idempotents of a group algebra and similar items involving only elements of certain conjugate subgroups.

For nonnormal subgroups, Theorem 1 is not especially pretty. The second theorem gives a more appealing inequality for the nonnormal case.

Applications of these results to matrix functions improve and unify earlier results of Williamson [10], Merris [7], Freese [4], and Merris and Watkins [8].

2. Relations in the group algebra. Let $H$ be a subgroup of the finite group $G$. Let $\chi$ be a (not necessarily irreducible) complex character of $H$. By $K G$ we mean the complex group algebra of $G$. Define

$$
t(H, \chi)=\frac{\chi(1)}{o(H)} \sum_{h \in H} \chi(h) h \in K G,
$$

where $o(H)$ is the order of $H$. Denote by $I(H)$ the set of irreducible complex characters on $H$. We know that $\{t(G, \eta): \eta \in I(G)\}$ is a set of mutually annihilating idempotents which spans $Z(K G)$, the center of $K G$ [1, p. 83], [2, pp. 233-236]. (Moreover, $t(G, \eta)$ generates the simple two sided ideal in $K G$ to which $\eta$ corresponds, and $\sum_{r \in I(G)} t(G, \eta)=$ $1 \in G$.)

THEOREM 1. Let

$$
L=\left\{g \in N_{G}(H): \chi\left(g^{-1} h g\right)=\chi(h): \forall h \in H\right\} .
$$

Let $g_{1}, \cdots, g_{r}$ be right coset representatives for $L$ in $G$. Let $\chi_{i}$ be 
the character on $g_{i}^{-1} H g_{i}$ defined by $\chi_{i}\left(g_{i}^{-1} h g_{i}\right)=\chi(h)$ for all $h \in H$. Then

$$
\frac{1}{r} \sum_{i=1}^{r} t\left(g_{i}^{-1} H g_{i}, \chi_{i}\right)=\sum_{\eta \in I(G)} \frac{\chi(1)(\chi, \eta)_{H}}{\eta(1)} t(G, \eta),
$$

where $(\chi, \eta)_{H}$ is the inner product,

$$
(\chi, \eta)_{H}=\frac{1}{o(H)} \sum_{h \in H} \chi(h) \eta\left(h^{-1}\right) .
$$

Proof. Let

$$
t=\sum_{i=1}^{r} t\left(g_{i}^{-1} H g_{i}, \chi_{i}\right)
$$

We first show that $t \in Z(K G)$. Let $g \in G$. Then

$$
g^{-1} t g=\frac{\chi(1)}{o(H)} \sum_{i=1}^{r} \sum_{h=H} \chi(h) g^{-1} g_{i}^{-1} h g_{i} g \text {. }
$$

Now, $L g_{i} \rightarrow L g_{i} g$ is a permutation of $\left\{L g_{i}: 1 \leqq i \leqq r\right\}$. Say $L g_{i} g=$ $L g_{\sigma(i)}$. Thus, there exists $l_{i} \in L$ such that $g_{i} g=l_{i} g_{\sigma(i)}, 1 \leqq i \leqq r$. Equation (2) becomes

$$
\begin{aligned}
g^{-1} t g & =\frac{\chi(1)}{o(H)} \sum_{i=1}^{r} \sum_{h \in H} \chi(h) g_{\sigma(i)}^{-1} l_{i}^{-1} h l_{i} g_{\sigma(i)} \\
& =\frac{\chi(1)}{o(H)} \sum_{i=1}^{r} \sum_{h \in H} \chi\left(l_{i}^{-1} h l_{i}\right) g_{\sigma(i)}^{-1} l_{i}^{-1} h l_{i} g_{\sigma(i)} \\
& =\frac{\chi(1)}{o(H)} \sum_{i=1}^{r} \sum_{h \in H} \chi(h) g_{\sigma(i)}^{-1} h g_{\sigma(i)} \\
& =t .
\end{aligned}
$$

It follows that $t \in Z(K G)$. From our previous remarks, there exist complex numbers $\mathscr{A}_{\eta}, \eta \in I(G)$, such that

$$
t=\sum_{\eta \in I(G)} \mathscr{A}_{\eta_{i}} t(G, \eta) \text {. }
$$

Since the $t(G, \eta)$ are annihilating idempotents, it follows that

$$
t t(G, \eta)=\mathscr{A}_{\eta} t(G, \eta)
$$

We now view $t$ and $t(G, \eta)$ as linear operators on $K G$. For example, to obtain $t(g)$, just multiply $g$ on the left by $t$, i.e., $t(g)=t g$. Then, from (3),

$$
\mathscr{A}_{\eta}=\frac{\operatorname{tr}(t t(G, \eta))}{\operatorname{tr}(t(G, \eta))}
$$

Let $\rho$ be the character of the regular representation of $G$. Then 


$$
\operatorname{tr}(t(G, \eta))=\frac{\eta(1)}{o(G)} \sum_{g \in G} \eta(g) \rho(g)=\eta(1)^{2} .
$$

Similarly, compute

$$
\begin{aligned}
\mathscr{A}_{\eta} & =\frac{1}{\eta(1)^{2}} \operatorname{tr}(t t(G, \eta)) \\
& =\frac{\chi(1)}{\eta(1) o(H) o(G)} \operatorname{tr}\left(\sum_{i=1}^{r} \sum_{h \in H} \sum_{g \in G} \chi(h) \eta(g) g_{i}^{-1} h g_{i} g\right) \\
& =\frac{\chi(1)}{\eta(1) o(H) o(G)} \sum_{i=1}^{r} \sum_{h \in H} \sum_{g \in G} \chi(h) \eta\left(g_{i}^{-1} h^{-1} g_{i} g\right) \rho(g) \\
& =\frac{\chi(1)}{\eta(1) o(H)} \sum_{i=1}^{r} \sum_{h \in H} \chi(h) \eta\left(h^{-1}\right) \\
& =\frac{\chi(1) r}{\eta(1)}(\chi, \eta)_{H} .
\end{aligned}
$$

(We have used the fact that $\operatorname{tr}(g)=\rho(g)=0$ if $g \neq 1$, and $\rho(1)=o(G)$.)

REMARKs. Suppose $H$ is normal in $G$. Let $\xi$ be a character of $G$ and let $\chi=\xi \mid H$. Then $L=G, r=1$, and (1) becomes

$$
t(H, \chi)=\chi(1) \sum_{\eta \in I(G)} \frac{(\chi, \eta)_{H}}{\eta(1)} t(G, \eta)
$$

If $\xi$ happens to be an irreducible character of $G$, then

$$
\chi=e\left(\lambda_{1}+\cdots+\lambda_{m}\right),
$$

where $\lambda_{1}=\lambda$ is an irreducible component of $\chi$ and $\lambda_{2}, \cdots, \lambda_{m}$ are the inequivalent conjugates of $\lambda$. The integer $e$ is the index of ramification of $\xi$ with respect to $H[3, \mathrm{p}$. 53]. In this case, (4) becomes

$$
t(H, \chi)=\frac{\chi(1)}{\lambda(1)} e \sum t(G, \eta)
$$

where the summation is over those irreducible characters, $\eta$, of $G$ whose restriction to $H$ contains $\lambda$ as a component. Expression (5) reveals that $t(H, \chi)$ is essentially idempotent when $H \triangle G$ and $\chi \in I(G)$. (This can, of course, be verified directly.)

If $A$ and $B$ are positive semidefinite hermitian operators on an $n$ dimensional complex inner product space, then $A \geqq B$ means that $A-B$ is positive semidefinite.

Theorem 2. Let $H$ be a subgroup of $G$. Let $\chi$ be an irreducible character on $H$. Then

$$
t(H, \chi) \geqq \sum t(G, \eta)
$$


where the summation is over those irreducible characters of $G$ whose restriction to $H$ is a multiple of $\chi$.

Proof. With respect to the inner product on $K G$ which makes $G$ an o.n. basis, $t(H, \chi)$ and $t(G, \eta)$ are hermitian. Since they are idempotent, they are orthogonal projections and hence are positive semidefinite. It is proved in [7, Corollary 2.4] that $t(H, \chi) \geqq t(G, \eta)$ if and only if $\eta \mid H$ is a multiple of $\chi$. Since $t(G, \eta) \in Z(K G)$, and since any two of them annihilate each other, the result follows.

3. Applications to matrix functions. We now assume that $G$ is a subgroup of $S_{n}$, the symmetric group of degree $n$. We still take $\chi$ to be a character on the subgroup $H$ of $G$. Let $r$ be a fixed but arbitrary integer in $[1, n]$. For the generic $n$-square complex matrix $A=\left(a_{i j}\right)$, define

$$
\delta_{\chi}^{H}(A)=\frac{\chi(1)}{o(H)} \sum_{g \in H} \chi(g) E_{r}\left(a_{l g(1,}, \cdots, a_{n g(n)}\right),
$$

where $E_{r}$ is the $r$ th elementary symmetric function.

When $r=n, \delta_{\chi}^{H}$ is $\chi(1) / o(H)$ times the generalized matrix function, $d_{\chi}^{H}$, of Schur [4], [5], and [9]. (If $H=S_{n}$ and $\chi$ is the alternating character, $d_{\chi}^{H}=$ determinant. If $H=\{1\}$ and $\chi=1, d_{\chi}^{H}=h$, the product of the main diagonal elements. If $H=S_{n}$ and $\chi=1, d_{\%}^{H}=$ permanent.)

When $r=1, \delta_{z}^{H}$ is $\chi(1) / o(H)$ times the generalized trace function, $t_{\%}^{I I}$ [6]. (If $H=\{1\}$ and $\chi=1, t_{\alpha}^{H}=$ trace. If $H=\langle(1,2, \cdots, n)\rangle$ and $\chi=1, t_{\chi}^{H}(A)$ is the sum of the entries of $A$.)

The case for general $r$ has also been discussed. See, for example, [7].

CoRollary 1. Let $H$ be a normal subgroup of $G$. Let $\xi$ be an irreducible character of $G$ and $\chi=\xi \mid H$. Then

$$
\delta_{\chi}^{H}(A)=\frac{\chi(1) e}{\lambda(1)} \sum \delta_{\gamma_{j}^{G}}^{G}(A),
$$

where the notation and summation are as they were in (5).

REMaRKs. If $H=\{1\}$, then (7) becomes

$$
E_{r}\left(a_{11}, \cdots, a_{n n}\right)=\sum_{\eta \in I(G)} \delta_{r}^{G}(A) .
$$

When $r=n$, (8) was obtained by Freese [4, eq. (8)].

If

$$
H=G_{\xi}=\{g \in G:|\xi(g)|=\xi(1)\},
$$


and $r=n$, equation (7) was obtained in [8].

When $\xi \mid H$ remains irreducible, (7) is a significant generalization of a result of Williamson [10, Theorem 1]. He showed that if $\xi(1)=$ 1 , then

$$
d_{\xi}^{G}(A) \leqq[G: H] d_{\xi}^{H}(A)
$$

for all positive semidefinite hermitian $A$. Equation (7) shows what has been discarded to obtain (9). In a similar way, equation (7) improves some of the results in [7].

A more general corollary could be obtained using the full power of Theorem 1. For simplicity, we use only (5).

Proof of Corollary 1. We begin by obtaining an alternate expression for $\delta_{x}^{G}$. First, some notation: Let $\left\{P(g): g \in S_{n}\right\}$ be the standard representation of $S_{n}$ by $n$-square permutation matrices, i.e., the $i, j$ element of $P(g)$ is $\delta_{i g(j)}$. Let $P_{r}(g)$ be the $r$ th Kronecker power of $P(g)$. Let $D_{r, n}$ be the set of one-to-one functions from $\{1,2, \cdots, r\}$ into $\{1,2, \cdots, n\}$. (In particular, $D_{n, n}=S_{n}$.) Let $Q_{r, n}$ be the subset of $D_{r, n}$ of order preserving one-to-one functions, i.e., $Q_{r, n}$ is the set

$$
\{\alpha=(\alpha(1), \cdots, \alpha(r)): 1 \leqq \alpha(1)<\alpha(2)<\cdots<\alpha(r) \leqq n\} .
$$

Now, observe

$$
\begin{aligned}
r ! \frac{o(H)}{\chi(1)} \delta_{\chi}^{H}(A) & =r ! \sum_{g \in H} \chi(g) \sum_{\alpha \in Q_{r n}} \prod_{i=1}^{r} a_{\alpha(i) g \alpha(i)} \\
& =\sum_{g \in H} \chi(g) \sum_{\alpha \in D_{r n}} \prod_{i=1}^{r} a_{\alpha(i) g \alpha(i)} \\
& =\sum_{g \in H} \chi(g) \sum_{\alpha, \beta \in D_{r n}} \prod_{i=1}^{r}\left(\delta_{\beta(i) g \alpha(i)} a_{\alpha(i) \beta(i)}\right) \\
& =\sum_{g \in H} \chi(g) \sum_{\alpha, \beta \in D_{r n}} \prod_{i=1}^{r}\left(P(g)_{\beta(i) \alpha(i)} a_{\alpha(i) \beta(i)}\right) \\
& =\sum_{g \in H} \chi(g) \sum_{\alpha, \beta \in D_{r n}}\left(P_{r}(g)_{\beta \alpha} K_{r}(A)_{\alpha \beta}\right) \\
& =\operatorname{trace}\left(C_{r}^{\prime}(H, \chi) K_{r}^{\prime}(A)\right),
\end{aligned}
$$

where $K_{r}(A)$ is the $r$ th Kronecker power of $A$,

$$
C_{r}(H, \chi)=\sum_{g \in H} \chi(g) P_{r}(g),
$$

and primes indicate the principal submatrices corresponding to $D_{r, n}$.

Now, $g \rightarrow P_{r}(g)$ is a representation of $S_{n}$. Thus, we may extend it linearly to a homomorphism, $\bar{P}_{r}$ of $K S_{n}$. To complete the proof, apply $\bar{P}_{r}$ to equation (5), restrict the resulting equation to the principal submatrices corresponding to $D_{r, n}$, and exploit the linearity of the expression (10). 
A similar corollary is available for Theorem 2. Since the proof is very similar, we will omit it.

COROLlaRY 2. We have

$$
\delta_{\chi}^{H}(A) \geqq \sum \delta_{\eta}^{G}(A)
$$

for all positive semidefinite hermitian $A$. The notation and summation are as they were in Theorem 2.

\section{REFERENCES}

1. Hermann Boener, Representations of Groups, American Elsevier, New York, 1970.

2. Charles W. Curtis and Irving Reiner, Representation Theory of Finite Groups and Associative Algebras, Interscience, New York, 1962.

3. Walter Feit, Characters of Finite Groups, Benjamin, New York, 1967.

4. Ralph Freese, Inequalities for generalized matrix functions based on arbitrary characters, preprint.

5. Marvin Marcus and Henryk Minc, Generalized matrix functions, Trans. Amer. Math. Soc., 116 (1965), 316-329.

6. Russell Merris, Trace functions I, Duke Math. J., 38 (1971), 527-530.

7. - A dominance theorem for partitioned hermitian matrices, Trans. Amer. Math. Soc., 164 (1972), 341-352.

8. Russell Merris and William Watkins, Character induced subgroups, J. Research Natl. Bur. Stds. 77B (1973), 93-99.

9. I. Schur, Über endliche Gruppen und Hermitesche Formen, Math. Z., 1 (1918), 184-207.

10. S. G. Williamson, On a class of combinatorial inequalities, J. Combinatorial Theory, 6 (1969), 359-369.

Received October 4, 1972.

California State University, Hayward 


\section{PACIFIC JOURNAL OF MATHEMATICS}

\section{EDITORS}

RICHARD ARENS (Managing Editor)

University of California

Los Angeles, California 90024

\section{R. A. Beaumont \\ University of Washington \\ Seattle, Washington 98105}

\section{J. DugundjI*}

Department of Mathematics University of Southern California Los Angeles, California 90007

D. Gilbarg and J. Milgram

Stanford University

Stanford, California 94305

\section{ASSOCIATE EDITORS}
E. F. BeCKenBaCH
B. H. NeumanN
F. WOLF
K. YoSHIDA

\section{SUPPORTING INSTITUTIONS}

\author{
UNIVERSITY OF BRITISH COLUMBIA \\ CALIFORNIA INSTITUTE OF TECHNOLOGY \\ UNIVERSITY OF CALIFORNIA \\ MONTANA STATE UNIVERSITY \\ UNIVERSITY OF NEVADA \\ NEW MEXICO STATE UNIVERSITY \\ OREGON STATE UNIVERSITY \\ UNIVERSITY OF OREGON \\ OSAKA UNIVERSITY
}

\author{
UNIVERSITY OF SOUTHERN CALIFORNIA \\ STANFORD UNIVERSITY \\ UNIVERSITY OF TOKYO \\ UNIVERSITY OF UTAH \\ WASHINGTON STATE UNIVERSITY \\ UNIVERSITY OF WASHINGTON

$* * *$
$*$
AMERICAN MATHEMATICAL SOCIETY
NAVAL WEAPONS CENTER

The Supporting Institutions listed above contribute to the cost of publication of this Journal, but they are not owners or publishers and have no responsibility for its content or policies.

Mathematical papers intended for publication in the Pacific Journal of Mathematics should be in typed form or offset-reproduced, (not dittoed), double spaced with large margins. Underline Greek letters in red, German in green, and script in blue. The first paragraph or two must be capable of being used separately as a synopsis of the entire paper. Items of the bibliography should not be cited there unless absolutely necessary, in which case they must be identified by author and Journal, rather than by item number. Manuscripts, in duplicate if possible, may be sent to any one of the four editors. Please classify according to the scheme of Math. Rev. Index to Vol. 39. All other communications to the editors should be addressed to the managing editor, or Elaine Barth, University of California, Los Angeles, California, 90024.

100 reprints are provided free for each article, only if page charges have been substantially paid. Additional copies may be obtained at cost in multiples of 50 .

The Pacific Journal of Mathematics is issued monthly as of January 1966. Regular subscription rate: $\$ 60.00$ a year (6 Vols., 12 issues). Special rate: $\$ 30.00$ a year to individual members of supporting institutions.

Subscriptions, orders for back numbers, and changes of address should be sent to Pacific Journal of Mathematics, 103 Highland Boulevard, Berkeley, California, 94708.

PUBLISHED BY PACIFIC JOURNAL OF MATHEMATICS, A NON-PROFIT CORPORATION

Printed at Kokusai Bunken Insatsusha (International Academic Printing Co., Ltd.), 270, 3-chome Totsuka-cho, Shinjuku-ku, Tokyo 160, Japan

* C. R. DePrima California Institute of Technology, Pasadena, CA 91109, will replace J. Dugundji until August 1974. 


\section{Pacific Journal of Mathematics}

Vol. 50, No. $2 \quad$ October, 1974

Mustafa Agah Akcoglu, John Philip Huneke and Hermann Rost, A counter example to the Blum Hanson theorem in general spaces .............

Huzihiro Araki, Some properties of modular conjugation operator of von

Neumann algebras and a non-commutative Radon-Nikodym theorem

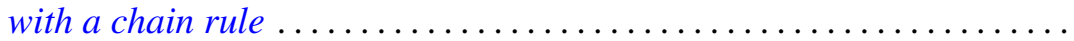

E. F. Beckenbach, Fook H. Eng and Richard Edward Tafel, Global properties of rational and logarithmico-rational minimal surfaces .....

David W. Boyd, A new class of infinite sphere packings ............. 383

K. G. Choo, Whitehead Groups of twisted free associative algebras ........

Charles Kam-Tai Chui and Milton N. Parnes, Limit sets of power series

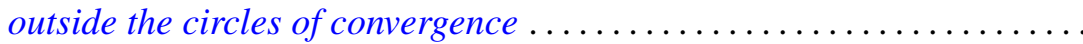

Allan Clark and John Harwood Ewing, The realization of polynomial

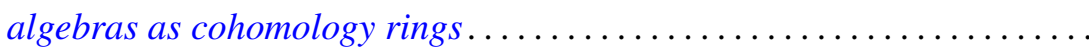

Dennis Garbanati, Classes of circulants over the p-adic and rational

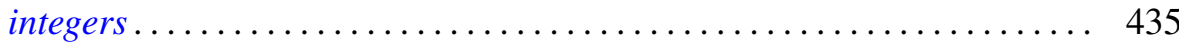

Arjun K. Gupta, On a "square" functional equation ................... 449

David James Hallenbeck and Thomas Harold MacGregor, Subordination and extreme-point theory ............................. 455

Douglas Harris, The local compactness of $v X \ldots \ldots . . . . . . . . . . . .4469$

William Emery Haver, Monotone mappings of a two-disk onto itself which fix the disk's boundary can be canonically approximated by homeomorphisms .................................. 477

Norman Peter Herzberg, On a problem of Hurwitz .................. 485

Chin-Shui Hsu, A class of Abelian groups closed under direct limits and subgroups formation ............................... 495

Bjarni Jónsson and Thomas Paul Whaley, Congruence relations and multiplicity types of algebras.....................

Lowell Duane Loveland, Vertically countable spheres and their wild sets.

Nimrod Megiddo, Kernels of compound games with simple components ....

Russell L. Merris, An identity for matrix functions ........ . .

E. O. Milton, Fourier transforms of odd and even tempered distributions ...

Dix Hayes Pettey, One-one-mappings onto locally connected generalized continua

Mark Bernard Ramras, Orders with finite global dimension

Doron Ravdin, Various types of local homogeneity. .

George Michael Reed, On metrizability of complete Moore spaces ...

Charles Small, Normal bases for quadratic extensions ..

Philip C. Tonne, Polynomials and Hausdorff matrices.... . . 